\title{
Die finnisch-ugrischen Sprachen Russlands sollen Unterrichtssprachen werden!
}

Янош Пустаи: Анализ словарей школьной терминологии удмуртского языка. [Analyse der Wortverzeichnisse zur udmurtischen Terminologie von Schulfächern.] Terminologia scholaris. Analysis V. NH Collegium Fenno-Ugristarum. Badacsonytördemic 2015. $427 \mathrm{~S}$.

János Pusztay hat ein großes, von der EU finanziertes Projekt geleitet, dessen Ziel es ist, fünf finnischugrische Sprachen (Komi, Mari, Mokscha, Ersä und Udmurtisch) in Russland wieder zur Unterrichtssprache in verschiedenen Schulfächern zu machen. Dies gilt heute, in einer Situation, in der immer weniger Kinder zu Hause solide Sprachkenntnisse erhalten, als eine der wichtigsten und effektivsten Maßnahmen zur Wiederbelebung der finnisch-ugrischen Sprachen. Im Hinblick auf dieses Ziel wurden für jede der genannten Sprachen Wörterbücher zur Terminologie von zehn Schulfächern erstellt: Sprache, Literatur, Geschichte, Gesellschaftskunde, Geografie, Biologie, Chemie, Physik, Mathematik und Informationstechnologie. Im Rahmen des Projekts entstanden also insgesamt 50 kleinere terminologische Wörterbücher (ca. 4060 Seiten, jeweils mit Hunderten Termini). Schon dies ist eine bemerkenswerte Leistung zugunsten der Entwicklung der Sprache. Darüber hinaus hat Pusztay für jede der Sprachen noch eine Analyse der Termini durchgeführt, für die der Wortschatz aller erwähnten Schulfächer zusammengestellt und mit den entsprechenden Termini der anderen zum Projekt gehörenden Sprachen verglichen wurden. Die Termini wurden u. a. daraufhin analysiert, ob sie eigensprachlich sind oder ganz oder teilweise aus dem Russischen übernommen wurden.

Die Analysen zur Terminologie der Schulfächer im Mokscha, Ersä, Komi und Mari sind bereits in den Jahren 2013-15 erschienen. Der nun vorliegende Band über die udmurtische Terminologie der Schulfächer ist also der letzte des Projekts. Dass die Termini aller im Rahmen des Projekts behandelten Sprachen parallel mit den udmurtischen präsentiert werden, ist meines Erachtens der wichtigste Ertrag dieses Bandes. Im Übrigen ist die auf den Seiten 14-17 vorgestellte 
Gliederung der Termini in 70 Gruppen nach ihrer Herkunft im Hinblick auf die praktische Anwendung und die Arbeit der Terminologen allzu detailliert und schematisch. Von Bedeutung ist vor allem die Gliederung der Termini in eigensprachliche, aus dem Russischen übernommene und internationale. Ein interessantes Resultat des Vergleichs der Sprachen ist die Feststellung, dass es im Udmurtischen in vielen Bereichen mehr eigensprachliche Termini gibt als in den anderen Sprachen. Erwartungsgemäß ist die Anzahl eigensprachlicher Termini in den mordwinischen Sprachen geringer als in den anderen.

Die Zusammenstellung der Terminologie aller Bereiche in einem Band ermöglicht auch einen Vergleich der Situation in den verschiedenen Bereichen (S. 420-421). In den Fächern Sprachwissenschaft, Biologie, Gesellschaftskunde, Geografie und Literatur sind die Termini hauptsächlich eigensprachlich, in den anderen Fächern vorwiegend entlehnt oder international. Das ist natürlich zu erwarten. Die Begriffe, die man in diesen Fächern benötigt, existieren in der Sprache meist auch als allgemein verwendete Wörter. Dagegen werden Termini der Chemie, Physik, Mathematik und Geschichte in der Alltagssprache weniger häufig verwendet. In dem Projekt wurden die eigensprachlichen Termini berücksichtigt, die bereits in den 1920er und 1930er Jahren vorgeschlagen worden waren. $\mathrm{Da}$ es damals noch keine Informationstechnologie gab, handelt es sich bei einem großen Teil der Termini dieses Bereichs um Entlehnungen, doch der Anteil der eigensprachlichen Bezeichnungen liegt auch hier immerhin bei 22,2 \%. Die Terminologie der Informationstechnik wurde zumindest im Udmurtischen und im Mari bereits in früheren Projekten entwickelt (http://translatedby.com/you/anglo-russko-marijsko-udmurtskijit-slovar/into-udm/). In welchem Umfang diese genutzt wurden, bleibt offen. Es scheint Unterschiede zu geben. Beispielsweise nennt das vorliegende Wortverzeichnis für Kursor das direkt aus dem Russischen übernommene Wort курсор, während die erwähnte frühere Wortliste die eigensprachliche Bezeichnung возьматэт anführt.

Die Liste der Termini, die in den Wortlisten mehrerer Fächer, aber in unterschiedlicher Form begegnen, ist ausgesprochen nützlich (S. 417). Im Allgemeinen handelt es sich um ein und denselben Terminus, für den es also keine Varianten geben sollte. Z. B. wird für den Begriff Nationalisierung 
Die finnisch-ugrischen Sprachen Russlands sollen Unterrichtssprachen werden!

in der Wortliste des Fachs Geschichte nur das aus dem Russischen übernommene Wort национализаиия angegeben, in der Wortliste zur Gesellschaftskunde dagegen das eigensprachliche кунваньбуртон. In dieser Liste fehlen einige Termini. Beispielsweise wird für den Begriff in der Wortliste zur Sprachwissenschaft кылъёз, in der Wortliste zur Literaturwissenschaft кyapa герӟem sowie als Teil von Komposita noch das aus dem Russischen übernommene слог angeführt. Es wäre angebracht, die Vereinheitlichung der Terminologie noch eingehender zu erörtern.

Im Hinblick auf das Gesamtprojekt, d.h. die Einführung eigensprachlichen Unterrichts auch in anderen Schulfächern als der Muttersprache, lassen das Vorwort und die Schlussworte des Buches zu wünschen übrig. Wie Pusztay erwähnt, handelt es sich vor allem um eine politische Frage. Er gibt auch zu verstehen, dass die (vorzugsweise eigensprachliche) Terminologie ausgefeilt, eigensprachliche Lehrbücher verfasst und die Lehrkräfte geschult werden müssen, bevor eigensprachlicher Unterricht angeboten werden kann. Dies sind selbstverständlich gute und erstrebenswerte Ziele. Die Terminologie kann jedoch nicht allzu weit entwickelt werden, ohne dass man sie in der Praxis, u. a. im Schulunterricht, erprobt.

In weiten Kreisen Udmurtiens wird udmurtischsprachiger Schulunterricht als Utopie betrachtet. Diese Auffassung teilen sogar einige Verfasser der in dem vorliegenden Band enthaltenen Wortverzeichnisse. In diesem Zusammenhang erscheinen die von Pusztay erwähnten weiteren Schritte wie in Russland erfundene Ausreden, die erklären sollen, dass eigensprachlicher Unterricht noch nicht verwirklicht werden könne. Im Unterricht ist die Hauptsache ja nicht die Terminologie, sondern das Verständnis der Inhalte. Besonders in den ersten Klassen, in denen der eigensprachliche Unterricht vermutlich zuerst eingeführt werden sollte, braucht man nicht allzu viele wissenschaftliche Termini. Die udmurtische Sprache und Literatur wurde in der gesamten zweiten Hälfte des 20. Jahrhunderts auf Udmurtisch unterrichtet, auch wenn damals fast alle Termini direkt aus dem Russischen übernommen wurden. Der Mangel an Termini war damals kein Hindernis für den Unterricht, warum also sollte er es jetzt sein. Unterricht ist zudem mehr als ein Lehrbuch. Es ist ohne weiteres möglich, den Unterricht auf Udmurtisch zu erteilen, wobei der Lehrer sein eigenes udmurtisches Material verwendet 
und die Schüler die verfügbaren wirkliches Hindernis für die Einrussischsprachigen Lehrbücher führung eines umfassenden Schulbenutzen. Kreative Lösungen die- unterrichts in udmurtischer Spraser Art sind an mehrsprachigen che besteht nicht. Die Hindernisse Schulen in der ganzen Welt üb- sind geistiger und politischer Art. lich. In den Dörfern sind auch die Lehrkräfte der verschiedenen FäEsa-Jussi Salminen cher schon jetzt sprachkundig. Ein 